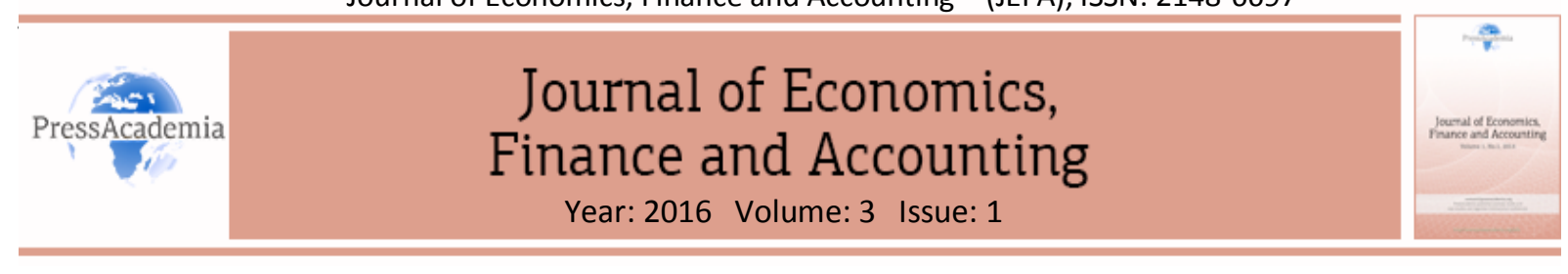

\title{
USING OF NET PRESENT VALUE (NPV) TO TEST THE INTEGRATED MODEL IN BUILDING MANAGEMENT INFORMATION SYSTEMS
}

\section{DOI: 10.17261/Pressacademia.2016116535}

\author{
Mohammad M M Abu Omar ', Khairul Anuar Abdullah² \\ 'Limkokwing University of Creative Technology -Malaysia, mmdabuomar@yahoo.com \\ ${ }^{2}$ Limkokwing University of Creative Technology - Malaysia, khairulanuarabdullah@limkokwing.edu.my
}

\begin{abstract}
The integrated model is a new model that is recently developed in order to build the management information systems (MIS's) by using the classical approach system development methodology. The integrated model aims to address the drawbacks of the classical approach in consumption additional time and cost while building the MIS's. The integrated model was subjected to two tests by using the mathematical probability theories in order to ensure the validity of the integrated model in its missio. The tests results have recorded the success of the integrated model in its mission. These tests are considered as internal tests, and thus, the integrated model still needs to an external practical test, in order to achieve a complete testing system for the integrated model, which will increase the confidence of the systems builders in using the integrated model. From here, this research uses the theory of net present value (NPV) financial function to implement a theoretical-practical test in order to test the validity of the integrated model in its work and mission.
\end{abstract}

Keywords: Management information system, MIS, integrated model, net present value, NPV. JEL Classification:

\section{INTRODUCTION}

The integrated model is a new model that is developed recently by Mohammad Abu Omar and Dr.Khairul Anuar Abdullah (Omar \& Abdullah, 2015a) in order to build the management information systems (MIS's) by using the classical approach, which is one of the systems development methodologies. The integrated model aims to overcome the drawbacks of the classical approach in consumption additional time and cost while building the (MIS's)(Omar \& Abdullah, 2015a).

The drawbacks of the classical approach in building the (MIS's) come from the permanent use of all stages of the information system life cycle (ISLC) that is adopted by classical approach; because in many cases, the building of (MIS's) by using the classical approach doesn't need the use of all stages in the (ISLC) that is adopted by classical approach, and this makes the classical approach consumes additional time and cost without really needing it(Omar \& Abdullah, 2015a).

The integrated model addresses the classical approach drawbacks through its work, that can be summarized as in the following (Omar \& Abdullah, 2015a):

A. The integrated model finds a new classification of the management problems that may be solved by building the MIS.

B. According to the new classification of the management problems, the integrated model develops new subapproaches from the classical approach to solve some of these management problems, without using all stages in the (ISLC) that is adopted by classical approach, and this avoids the classical approach to consuming additional time and cost while building the (MIS's) to solve the management problems. 


\subsection{The Management Problems Classification in the Integrated Model}

The integrated model classifies the management problems that may be solved by building the (MIS's) into three main categories, as follows (Omar \& Abdullah, 2015a, b):

- First category management problems: this category includes to the following management problems:

a- First order management problems.

b- Second order management problems.

- Second category management problems: this category includes to the following management problems:

a- First level management problems.

b- Second level management problems.

- Combined management problems: this category includes to the following management problems:

a- First combined management problems.

b- Second combined management problems.

c- Third combined management problems.

d- Fourth combined management problems.

Each one of the previous management problems has its special nature and characteristics.

\subsection{The Developed Sub-Approaches in the Integrated Model}

The classical approach adopts in its work the information system life cycle (ISLC)(Aktas, 1987), which consists of the series of defined stages or phases, which should occur when building the MIS These stages are:Planing Stage, Analysis Stage, Design Stage, Development\& Programing Stage, and Test \&Maintenance Stage,as depicted in the following figure(Aktas, 1987),(Omar \& Abdullah, 2015a):

Figure 1: The Information System Life Cycle (ISLC) of Classical Approach.

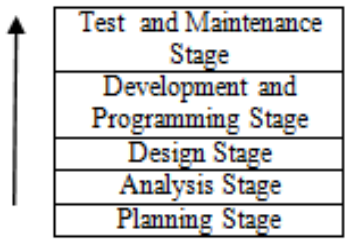

The integrated model develops new sub-approaches from the classical approach life cycle which have a minimized life cycle compared with the current classical approach life cycle, these sup-approaches are used to solve some of the management problems that are found by the integrated model in order to save the time and cost when using the classical approach to build the (MIS's) to solve the management problems. These new subapproaches are as follows (Omar \& Abdullah, 2015a):

- MIS New Approach (1):

This sub-approach is developed from the classical approach life cycle to solve some of the management problems without consumption additional time and cost, these problems are: first order management problems and second combined management problems. According to the nature and characteristics of these management problems(Omar \& Abdullah, 2015a), the MIS new approach (1) skips the second stage (Analysis Stage) in the information system life cycle (ISLC) that is adopted by the classical approach, and thus, the MIS new approach (1) uses four stages instead of five stages, as in the following figure(Omar \& Abdullah, 2015a): 
Figure 2: The MIS New Approach (1)

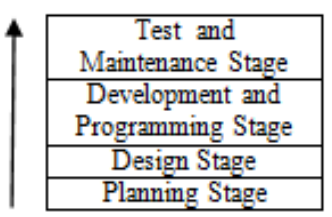

- MIS New Approach (2):

This sub-approach is developed from the classical approach life cycle to solve some of the management problems without additional consumption in time and cost, these problems are: first level management problems and third combined management problems. And according to the nature and characteristics of these management problems(Omar \& Abdullah, 2015a) the MIS new approach (2) skips the third stage (Design Stage) in the information system life cycle (ISLC) that is adopted by the classical approach, and thus, the MIS new approach (2) uses four stages instead of five stages, as in the following figure(Omar \& Abdullah, 2015a):

Figure 3: The MIS New Approach (2)

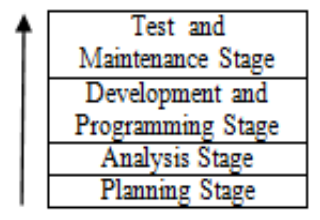

- MIS New Approach (3):

This sub-approach is developed from the classical approach life cycle to solve one of the management problems without additional consumption in time and cost, these problems are: first combined management problems. And according to the nature and characteristics of these management problems (Omar \& Abdullah, 2015a), the MIS new approach (3) skips the both second stage (Analysis Stage) and third stage (Design Stage) in the information system life cycle (ISLC) that is adopted by the classical approach, and thus, the MIS new approach (3) uses three stages instead of five stages, as in the following figure(Omar \& Abdullah, 2015a):

Figure (4): The MIS New Approach (3)

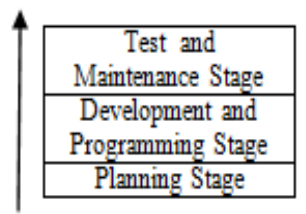

The integrated model stays the use of the current approach of a classical approach to solve the other management problems that are not solved by the previous three developed sub-approaches. The current approach of the classical approach is named as MIS classical approach, as in the following figure (Omar \& Abdullah, 2015a):

Figure 5: The MIS Classical Approach

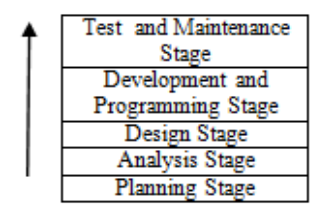


The following figure shows the integrated model structure and work (Omar \& Abdullah, 2015a):

Figure 5: The Integrated Model Structure and Work

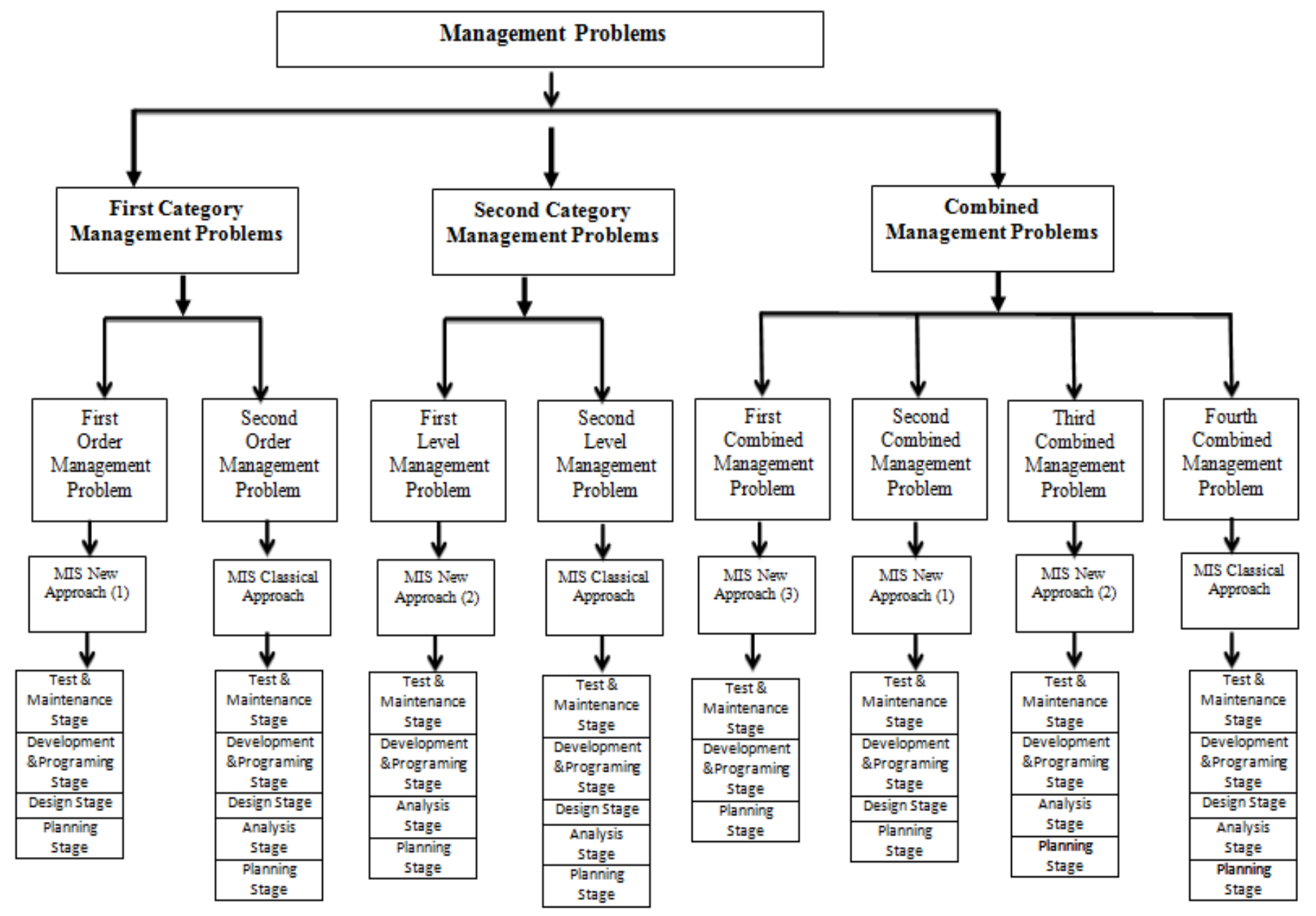

The use of this integrated model has been tested by using the mathematical probability theories, to prove how efficiently and successfully the model works, and the tests results confirm the success of the integrated model in its work (Omar \& Abdullah, 2015b), but these tests are considered as internal tests, so, the integrated model still needs to an external practical test, which will increase the confidence of system-developers in the integrated model, accordingly, this paper uses the net present value (NPV) to implement a theoretical practical test to the integrated model.

\section{RESEARCH METHODOLOGY}

The integrated model was subjected to the evaluation through two tests, which are:

A- Testing the integrated model by using the simple random sampling (SRS)probability theory(Mohammad Abu Omar and Khairul Anuar,2015). This test is implemented to examine if the integrated model can achieve its mission in solving the management problems through the building of the (MIS's) by using the classical approach 
without consumption additional time and cost, the tests results show the success of the integrated model in its work with the percentage of $62.5 \%$.

B- Testing the integrated model by using the probability tree diagram (Mohammad Abu Omar and Khairul Anuar,2016). This test is implemented because it gives more accurate results compared with the simple random sampling probability test, and the test results have recorded a new success of the integrated model work with the percentage of more than $58 \%$.

The previous tests are considered as internal tests[2],[6], and there is a recommendation for future work to implement an external theoretical test which is a practical test(Omar \& Abdullah, 2015a),(Omar \& Abdullah, 2015b), in order to increase the confidence of the integrated model work, and from here, this research is done in order to implement this theoretical practical test.

This research uses the theory of the net present value (NPV) financial function to implement a theoretical practical test to the integrated model. The net present value (NPV) helps the investors to invest in the best project, because the NPV provides a comparison between the offered projects, and this helps the investors to invest in the best project. The NPV is defined as the difference between the present value of cash inflows and the present value of cash outflows, the NPV can also be defined as the difference between the present value of benefits (PVB) and the present value of costs (PVC) (Brealey, 2012). In the following is the structure and analysis of the NPV mathematical formula:

The present value of cash benefits (inflows): $\quad P V B=\sum_{t=0}^{n} \frac{B_{t}}{(1+r)^{t}}$

Where:

t: Time.

$\mathrm{n}$ : The life of the project.

$\mathrm{B}_{\mathrm{t}}$ : Are the benefits in year $\mathrm{t}$.

$r$ : The discount rate.

$\frac{1}{(1+r)^{t}}$ : The discount factor.

The present value of cash costs (outflows): $\quad P V C=\sum_{t=0}^{n} \frac{C_{t}}{(1+r)^{t}}$

Where:

$\mathrm{t}$ : Time.

$\mathrm{n}$ : The life of the project.

$C_{t}$ : Are the costs in year $t$.

$r$ : The discount rate.

$\frac{1}{(1+r)^{t}}$ : The discount factor.

And therefore, the NPV= PVB $-\mathrm{PVC}$

So, the net present value (NPV) $=\sum_{t=0}^{n} \frac{B_{t}-C_{t}}{(1+r)^{t}}$

And thus, the NPV $=B_{0}-C_{0}+\frac{B_{1}-C_{1}}{(1+r)}+\frac{B_{2}-C_{2}}{(1+r)^{2}}+\frac{B_{3}-C_{3}}{(1+r)^{3}}+\ldots \ldots+\frac{B_{n}-C_{n}}{(1+r)^{n}}$

Where:

$\mathrm{B}_{0}$ : Are the benefits at the beginning of the project (at year 0 ). So, $\mathrm{B}_{0}$ is usually $=0$.

$\mathrm{C}_{0}$ : Are the cost at the beginning of the project (at year 0 ), which are known as: the initial cost of the investment.

$B_{t}-C_{t}$ : Are the cash flow (net cash flow) at year $t$. 
Now, and by substituting $B_{0}=0$, the NPV will be as is follows:

The NPV $=-C_{0}+\frac{B_{1}-C_{1}}{(1+r)}+\frac{B_{2}-C_{2}}{(1+r)^{2}}+\frac{B_{3}-C_{3}}{(1+r)^{3}}+\ldots \ldots .+\frac{B_{n}-C_{n}}{(1+r)^{n}}$

Hence, the NPV formula can be written as is follows:

The NPV $=\sum_{t=0}^{n} \frac{B_{t}-C_{t}}{(1+r)^{t}}-C_{0}$

And, in order to obtain the general mathematical formula to the NPV; the $\left(B_{t^{-}} C_{t}\right)$ will be substituted by $\left(F_{t}\right)$, as in the following:

The NPV $=\sum_{t=0}^{n} \frac{F_{t}}{(1+r)^{t}}-C_{0}=-C_{0}+\frac{F_{1}}{(1+r)}+\frac{F_{2}}{(1+r)^{2}}+\frac{F_{3}}{(1+r)^{3}}+\ldots \ldots+\frac{F_{n}}{(1+r)^{n}}$

Where:

$\mathrm{t}$ : Time.

$\mathrm{n}$ : The life of the project.

$F_{t}$ : Are the net cash flow in year $t$.

$r$ : The discount rate.

$\frac{1}{(1+r)^{t}}$ : The discount factor.

$C_{0}$ : Are the cost at the beginning of the project (at year 0 ), which are known as: the initial cost of the investment.

The NPV theory in evaluation of the projects states that the project will be accepted for investment, if and only if the NPV of this project is greater than zero,(NPV>0), and in the cases of NPV is smaller or equal to zero (NPV<= 0), the project will be rejected for investment(Brealey, 2012),

If there are two projects, and there is a need to choose one of them for the investment, and both of them have a value of NPV which is greater than zero (NPV $>0)$, the project of the highest NPV value will be chosen(Brealey, 2012).

This research will apply the NPV theory as a practical theoretical test to the integrated model as in the following procedure:

Step1: Apply the general mathematical formula of NPV on the project which uses the (ISLC) of the classical approach, which the integrated model defines it as: MIS classical approach.

Step2: Apply the general mathematical formula of NPV on the following projects:

a- The project which uses the developed sub-approach: MIS new approach(1), that is developed by the integrated model.

b- The project which uses the developed sub-approach: MIS new approach(2), that is developed by the integrated model.

c- The project which uses the developed sub-approach: MIS new approach(3), that is developed by the integrated model.

Step3: Implement a comparison between the results of the previous two steps by using the theory of NPV; to test the validity of the integrated model in its work. 
Now, the steps(1and 2) will be implemented through the following example, and the step(3), will be implemented in the section(4).

\section{Application Example:}

A company is seeking to invest in one of the projects, the period of the investment is ( $n$ ) years. According to this investment, there is an initial cost of the investment which is the cost of building the management information system (MIS), and also, there will be the net cash flows through the years of the investment.

\section{- The implementation of (step 1) on the application example:}

Assuming that the company will build the required MIS by using the (ISLC) that is adopted by the classical approach, that is defined by the integrated model as: MIS classical approach. In this case, the building process of the (MIS) will be done through the sequential stages of the (ISLC) that is adopted by classical approach, which is as follows:

Figure 6: Information System Life Cycle (ISLC) of Classical Approach, (MIS Classical Approach).

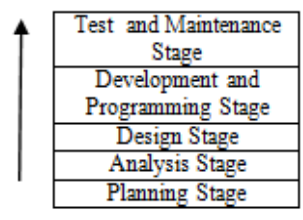

So, the initial costs of the investment in this projects, which are the costs of building the required (MIS), will be the summation of the cost in each stage of the (ISLC). Now, assuming that the cost in each stage of the MIS classical approach, as follows:

- The cost of planning stage is: $X_{1}$

- The cost of analysis stage is: $X_{2}$

- The cost of design stage is: $X_{3}$

- The cost of development\& programing stage is: $X_{4}$

- The cost of maintenance stage is: $X_{5}$

And thus, the initial cost $=\left(X_{1}+X_{2}+X_{3}+X_{4}+X_{5}\right)$

And the general formula of the NPV will now be applied, as is follows:

The NPV $=\sum_{t=0}^{n} \frac{F_{t}}{(1+r)^{t}}-C_{0}=-C_{0}+\frac{F_{1}}{(1+r)}+\frac{F_{2}}{(1+r)^{2}}+\frac{F_{3}}{(1+r)^{3}}+\ldots \ldots+\frac{F_{n}}{(1+r)^{n}}$

And by substituting the initial cost $\left(C_{0}\right)$ by: $\left(X_{1}+X_{2}+X_{3}+X_{4}+X_{5}\right)$, the NPV general formula will be defined as (NPV 1), as in the following equation(1):

The NPV $1=-\left(X_{1}+X_{2}+X_{3}+X_{4}+X_{5}\right)+\frac{F_{1}}{(1+r)}+\frac{F_{2}}{(1+r)^{2}}+\frac{F_{3}}{(1+r)^{3}}+\ldots \ldots .+\frac{F_{n}}{(1+r)^{n}}$

\section{- The implementation of (step 2- a) on the application example:}

Assuming that the company will build the required (MIS) by using the developed sub-approach: MIS new approach(1), that is developed by the integrated model. In this case, the building process of the (MIS) will be done through the sequential stages of the MIS new approach(1), which are as follows: 
Figure 7: The MIS New Approach (1)

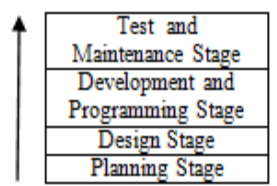

So, the initial cost of the investment in this projects, which are the cost of building the required (MIS), will be the summation of the cost in each stage of the MIS new approach(1). Now, assuming Now, assuming that the cost in each stage of the MIS new approach(1), as follows:

- The cost of planning stage is: $\mathrm{X}_{1}$

- The cost of analysis stage is: $X_{2}=0$; (Because it is skipped by MIS new approach(1)).

- The cost of design stage is: $\mathrm{X}_{3}$

- The cost of development\& programing stage is: $\mathrm{X}_{4}$

- The cost of maintenance stage is: $x_{5}$

And thus, the initial cost $=\left(X_{1}+X_{3}+X_{4}+X_{5}\right)$

And the general formula of the NPV will now be applied, as follows:

The NPV $=\sum_{t=0}^{n} \frac{F_{t}}{(1+r)^{t}}-C_{0}=-C_{0}+\frac{F_{1}}{(1+r)}+\frac{F_{2}}{(1+r)^{2}}+\frac{F_{3}}{(1+r)^{3}}+\ldots \ldots .+\frac{F_{n}}{(1+r)^{n}}$

And by substituting the initial costs $\left(C_{0}\right)$ by: $\left(X_{1}+X_{3}+X_{4}+X_{5}\right)$, the NPV general formula will be defined as (NPV $2)$, as in the following equation(2):

The NPV $2=-\left(X_{1}+X_{3}+X_{4}+X_{5}\right)+\frac{F_{1}}{(1+r)}+\frac{F_{2}}{(1+r)^{2}}+\frac{F_{3}}{(1+r)^{3}}+\ldots \ldots+\frac{F_{n}}{(1+r)^{n}} \ldots .$. (2)

\section{- The implementation of (step 2- b) on the application example:}

Assuming that the company will build the required (MIS) by using the developed sub-approach: MIS new approach(2), that is developed by the integrated model. In this case, the building process of the (MIS) will be done through the sequential stages of the MIS new approach(1), which are as follows:

Figure 8: The MIS New Approach (2)

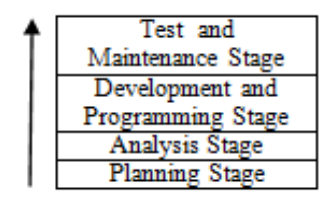

So, the initial cost of the investment in this projects, which are the costs of building the required (MIS), will be the summation of the cost in each stage of the MIS new approach(2). Now, assuming that the cost in each stage of the MIS new approach(2), as follows:

- The cost of planning stage is: $\mathrm{X}_{1}$

- The cost of analysis stage is: $X_{2}$

- The cost of design stage is: $\mathrm{X}_{3}=0$; (Because it is skipped by MIS new approach(2)). 
- The cost of development\& programing stage is: $\mathrm{X}_{4}$

- The cost of maintenance stage is: $X_{5}$

And thus, the initial cost $=\left(X_{1}+X_{2}+X_{4}+X_{5}\right)$

And the general formula of the NPV will now be applied, as is follows:

The NPV $=\sum_{t=0}^{n} \frac{F_{t}}{(1+r)^{t}}-C_{0}=-C_{0}+\frac{F_{1}}{(1+r)}+\frac{F_{2}}{(1+r)^{2}}+\frac{F_{3}}{(1+r)^{3}}+\ldots \ldots .+\frac{F_{n}}{(1+r)^{n}}$

And by substituting the initial cost $\left(C_{0}\right)$ by: $\left(X_{1}+X_{2}+X_{4}+X_{5}\right)$, the NPV general formula will be defined as (NPV $3)$, as in the following equation(3):

The NPV $3=-\left(X_{1}+X_{2}+X_{4}+X_{5}\right)+\frac{F_{1}}{(1+r)}+\frac{F_{2}}{(1+r)^{2}}+\frac{F_{3}}{(1+r)^{3}}+\ldots \ldots .+\frac{F_{n}}{(1+r)^{n}} \ldots \ldots .$. (3)

\section{- The implementation of (step 2- c) on the application example:}

Assuming that the company will build the required (MIS) by using the developed sub-approach: MIS new approach(3), that is developed by the integrated model. In this case, the building process of the (MIS) will be done through the sequential stages of the MIS new approach(3), which are as follows:

Figure8: The MIS New Approach (3)

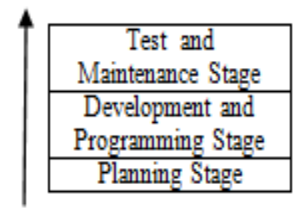

So, the initial cost of the investment in this projects, which are the cost of building the required (MIS), will be the summation of the cost in each stage of the MIS new approach(3). Now, assuming that the cost in each stage of the MIS new approach(3), as follows:

- The cost of planning stage is: $X_{1}$

- The cost of analysis stage is: $X_{2}=0$; (Because it is skipped by MIS new approach(3)).

- The cost of design stage is: $X_{3}=0$; (Because it is skipped by MIS new approach(3)).

- The cost of development\& programing stage is: $\mathrm{X}_{4}$

- The cost of maintenance stage is: $X_{5}$

And thus, the initial cost $=\left(X_{1}+X_{4}+X_{5}\right)$

And the general formula of the NPV will now be applied, as is follows:

The NPV $=\sum_{t=0}^{n} \frac{F_{t}}{(1+r)^{t}}-C_{0}=-C_{0}+\frac{F_{1}}{(1+r)}+\frac{F_{2}}{(1+r)^{2}}+\frac{F_{3}}{(1+r)^{3}}+\ldots \ldots+\frac{F_{n}}{(1+r)^{n}}$

And by substituting the initial cost $\left(C_{0}\right)$ by: $\left(X_{1}+X_{4}+X_{5}\right)$, the NPV general formula will be defined as (NPV 4$)$, as in the following equation(4): 
The NPV $4=-\left(X_{1}+X_{4}+X_{5}\right)+\frac{F_{1}}{(1+r)}+\frac{F_{2}}{(1+r)^{2}}+\frac{F_{3}}{(1+r)^{3}}+\ldots \ldots .+\frac{F_{n}}{(1+r)^{n}}$

\section{FINDINGS and DISCUSSION}

Here, this research implements a comparison between the expected values that may be resulted from the (NPV 1) from one side and the expected values that may result from the (NPV 2), (NPV 3), and (NPV 4), from the other side.

\subsection{The Comparision Between the Expected Values of (NPV 1) \& (NPV 2)}

The (NPV 1), is the NPV of the project which builds the management information system (MIS) by using the MIS classical approach, which is the (ISLC) that is adopted by the classical approach. And as it is mentioned in the research methodology, the (NPV 1) has the following equation:

The NPV $1=-\left(X_{1}+X_{2}+X_{3}+X_{4}+X_{5}\right)+\frac{F_{1}}{(1+r)}+\frac{F_{2}}{(1+r)^{2}}+\frac{F_{3}}{(1+r)^{3}}+\ldots \ldots .+\frac{F_{n}}{(1+r)^{n}}$

On the other hand, the (NPV 2), is the NPV of the project which builds the management information system (MIS) by using the MIS New approach(1), which is a new approach, that has been recently developed by the integrated model. And as it is mentioned in the research methodology, the (NPV 2) has the following equation:

The NPV $2=-\left(X_{1}+X_{3}+X_{4}+X_{5}\right)+\frac{F_{1}}{(1+r)}+\frac{F_{2}}{(1+r)^{2}}+\frac{F_{3}}{(1+r)^{3}}+\ldots \ldots .+\frac{F_{n}}{(1+r)^{n}}$

The mathematical comparison between the equations of (NPV 1) and (NPV 2) gives the following results:

a- The equation of the (NPV 1$)$ has the negative part: $-\left(X_{1}+X_{2}+X_{3}+X_{4}+X_{5}\right)$

b- The equation of the (NPV 2) has the negative part: - $\left(X_{1}+X_{3}+X_{4}+X_{5}\right)$

So, it is obviously that the negative part of the equation of (NPV 1 ) is larger than the negative part in the equation of (NPV 2), and this will lead to the following results and facts:

(NPV 2) > (NPV 1): The value of (NPV 2) is larger than the value of (NPV 1), this fact will lead to the following results according to the NPV theory:

1- The possibility that the (NPV 2) is larger than zero (NPV $2>0$ ) is higher than the possibility that the (NPV 1) is larger than zero. And hence, the possibility of the acceptance the project in case of (NPV 2) is higher than the possibility of the acceptance the project in case of (NPV 1), so, the project that uses the developed MIS new approach(1) will have a higher acceptance in the (NPV) test, than the project that uses the MIS classical approach.

2- In case that both of (NPV 1) and (NPV 2) have values larger than zero, the project in the case of (NPV 2) will be chosen, and not the project in the case of (NPV 1), on other hand, the project that uses the developed MIS new approach(1) will be chosen and not the project that uses the MIS classical approach.

And thus, the previous results confirm the surpass of the MIS new approach(1) compared with the MIS classical approach, in building the management information systems, on the other meaning, the results confirm the success of the integrated model in building the MIS's by using the developed MIS new approach(1). 


\subsection{The Comparision Between the Expected Values of (NPV 1) \& (NPV 3)}

The (NPV 1), is the NPV of the project which builds the management information system (MIS) by using the MIS classical approach, which is the (ISLC) that is adopted by the classical approach. And as it is mentioned in the research methodology, the (NPV 1) has the following equation:

The NPV $1=-\left(X_{1}+X_{2}+X_{3}+X_{4}+X_{5}\right)+\frac{F_{1}}{(1+r)}+\frac{F_{2}}{(1+r)^{2}}+\frac{F_{3}}{(1+r)^{3}}+\ldots \ldots .+\frac{F_{n}}{(1+r)^{n}}$

On the other hand, the (NPV 3), is the NPV of the project which builds the management information system (MIS) by using the MIS New approach(2), which is a new approach, that has been recently developed by the integrated model. And as it is mentioned in the research methodology, the (NPV 3 ) has the following equation:

The NPV $3=-\left(X_{1}+X_{2}+X_{4}+X_{5}\right)+\frac{F_{1}}{(1+r)}+\frac{F_{2}}{(1+r)^{2}}+\frac{F_{3}}{(1+r)^{3}}+\ldots \ldots .+\frac{F_{n}}{(1+r)^{n}}$

The mathematical comparison between the equations of (NPV 1) and (NPV 3) gives the following results:

a- The equation of the (NPV 1) has the negative part: $-\left(X_{1}+X_{2}+X_{3}+X_{4}+X_{5}\right)$

b- The equation of the (NPV 3) has the negative part: $-\left(X_{1}+X_{2}+X_{4}+X_{5}\right)$

So, it is obviously that the negative part of the equation of (NPV 1) is larger than the negative part in the equation of (NPV 3), and this will lead to the following results and facts:

(NPV 3) > (NPV 1): The value of (NPV 3) is larger than the value of (NPV 1), this fact will lead to the following results according to the NPV theory:

1- The possibility that the (NPV 3) is larger than zero (NPV $3>0$ ) is higher than the possibility that the (NPV 1) is larger than zero. Hence, the possibility of the acceptance the project in case of (NPV 3) is higher than the possibility of the acceptance the project in case of (NPV 1). Therefore, the project that uses the developed MIS new approach(2) will have a higher acceptance in the (NPV) test, than the project that uses the MIS classical approach.

2- In case that both of (NPV 1) and (NPV 3) have values larger than zero, the project in the case of (NPV 3) will be chosen, and not the project in the case of (NPV 1), on other hand, the project that uses the developed MIS new approach(2) will be chosen and not the project that uses the MIS classical approach.

And thus, the previous results confirm the surpass of the MIS new approach(2) compared with the MIS classical approach, in building the management information systems, on the other meaning, the results confirm the success of the integrated model in building the MIS's by using the developed MIS new approach(2).

\subsection{The Comparision Between the Expected Values of (NPV 1) \& (NPV 4)}

The (NPV 1), is the NPV of the project which builds the management information system (MIS) by using the MIS classical approach, which is the (ISLC) that is adopted by the classical approach. And as mentioned in the research methodology, the (NPV 1) has the following equation:

The NPV $1=-\left(X_{1}+X_{2}+X_{3}+X_{4}+X_{5}\right)+\frac{F_{1}}{(1+r)}+\frac{F_{2}}{(1+r)^{2}}+\frac{F_{3}}{(1+r)^{3}}+\ldots \ldots . .+\frac{F_{n}}{(1+r)^{n}}$

On the other hand, the (NPV 4), is the NPV of the project which builds the management information system (MIS) by using the MIS New approach(3), which is a new approach, that has been recently developed by the integrated model. And as it is mentioned in the research methodology, the (NPV 4) has the following equation: 


$$
\text { The NPV } 4=-\left(X_{1}+X_{4}+X_{5}\right)+\frac{F_{1}}{(1+r)}+\frac{F_{2}}{(1+r)^{2}}+\frac{F_{3}}{(1+r)^{3}}+\ldots \ldots .+\frac{F_{n}}{(1+r)^{n}}
$$

The mathematical comparison between the equations of (NPV 1) and (NPV 4) gives the following results:

a- The equation of the (NPV 1) has the negative part: $-\left(X_{1}+X_{2}+X_{3}+X_{4}+X_{5}\right)$

b- The equation of the (NPV 4) has the negative part: $-\left(X_{1}+X_{4}+X_{5}\right)$

So, it is obviously that the negative part of the equation of (NPV 1) is larger than the negative part in the equation of (NPV 4), and this will lead to the following results and facts:

(NPV 4) > (NPV 1): The value of (NPV 4) is larger than the value of (NPV 1), this fact will lead to the following results according to the NPV theory:

1- The possibility that the (NPV 4) is larger than zero (NPV $4>0$ ) is higher than the possibility that the (NPV 1) is larger than zero. And hence, the possibility of the acceptance the project in case of (NPV 4) is higher than the possibility of the acceptance the project in case of (NPV 1), so, the project that uses the developed MIS new approach(3) will have a higher acceptance in the (NPV) test, than the project that uses the MIS classical approach.

2- In case that both of (NPV 1) and (NPV 4) have values larger than zero, the project in the case of (NPV 4) will be chosen, and not the project in the case of (NPV 1). And on other hand, the project that uses the developed MIS new approach(3) will be chosen and not the project that uses the MIS classical approach.

And thus, the previous results confirm the superiority of the MIS new approach(3) compared with the MIS classical approach, in building the management information systems. Additionally, the results confirm the success of the integrated model in building the MIS's by using the developed MIS new approach(3).

\subsection{The Evaluation of the validity of the Integrated Model of Building the Management Information Systems}

The previous results of the NPV test, prove the superiority of all new approaches: MIS new approach(1), MIS new approach(2), and MIS new approach(3) on the MIS classical approach which uses the ISLC that is adopted by the classical approach. And because the new approaches: MIS new approach(1), MIS new approach(2), and MIS new approach(3) have been developed by the integrated model which uses them in building the MIS's in order to solve more than $62 \%$ of the management problems that are defined by the integrated model (Omar \& Abdullah, 2015b), so the research's results confirm the success and the validity of the integrated model in its work.

\section{CONCLUSION}

This research uses the theory of net present value (NPV) to implement a theoretical practical test for the integrated model, and the test results confirm the success and validity of the integrated model in improving the use of the classical approach in building the management information systems (MIS's). Since the research implements a comparison between building the (MIS) by using of the new sub-approaches that are developed by the integrated model which are: MIS new approach(1), MIS new approach(2), and MIS new approach(3), and building the (MIS) by using the (ISLC) that is adopted by the classical approach,and the comparison results show the superiority of the sub-approaches: MIS new approach(1), MIS new approach(2), and MIS new approach(3) that are developed by the integrated model, compared with the (ISLC) that is adopted by the classical approach. 


\section{REFERENCES}

Abu Omar, M. M. (2014). A New Approach to Increase the Efficiency of Classical Approach In Designing Management Information Systems (MIS'S). IJRCCT, 3(11), 1421-1424

Aktas, A. Z. (1987). Structured analysis \& design of information systems. Prentice-Hall, Inc.

Brazier, J. (2007). Measuring and valuing health benefits for economic evaluation. Oxford University Press.

Brealey, R. A., Myers, S. C., Allen, F., \& Mohanty, P. (2012). Principles of corporate finance. Tata McGraw-Hill Education.

Dixon, J. A., \& Hufschmidt, M. M. (1986). Economic evaluation techniques for the environment.

Hardcastle, E. (2011). Business Information Systems. Bookboon.

Hoy, M. (2001). Mathematics for economics: student's solutions manual. MIT press.

http://www.investopedia.com

MMAbuOmar, Mohammed. "Developing New Methods in designing Management Information Systems to solve Management Problems by using Classical Approach." International Journal of Computer Applications 101.16 (2014): 27-30.

Omar, M. M. A., \& Abdullah, K. A. (2015). Developing a New Integrated Model to improve the using of Classical Approach in Designing Management Information Systems. International Journal of Advanced Computer Science \& Applications, 1(6), 190197.

Omar, M. M. A., \& Abdullah, K. A.(2015). Testing the Use of the Integrated Model in Designing the Management Information Systems by Using the Mathematical Probability Theories. International Journal of Advanced Computer Science \& Applications, 1(6), 159-165.

Omar, M. M. A., \& Abdullah, K. A.(2016). The Use of the Probability Tree Digram to Test the Integrated Model in building the Management Information Systems.

Takayama, Akira. Mathematical economics. Cambridge University Press, 1985. 